\title{
Evaluation of two-dimensional strain echocardiography for quantifying right ventricular function in patients with pulmonary arterial hypertension
}

\author{
YONG LIU, YONG WANG, YINGYING WANG and ZHE WEN \\ Department of Ultrasound, Beijing Shijitan Hospital, Capital Medical University, Beijing 100038, P.R. China
}

Received January 31, 2016; Accepted February 2, 2017

DOI: $10.3892 /$ etm.2017.4581

\begin{abstract}
The aim of the current study was to evaluate the use of two-dimensional strain echocardiography (2DS) in quantifying right ventricular (RV) function in patients with pulmonary arterial hypertension (PAH). A total of 41 patients with PAH and 40 healthy control patients were recruited to the study. 2DS was used to determine the strain $(\varepsilon)$ and strain rate (SR) of basal, middle and apical segments of the RV free wall. The aforementioned indicators were compared with measurements of RV function taken by conventional ultrasound and right heart catheterization. The $\varepsilon$ and SR of all segments of the right ventricular free wall were significantly lower in the PAH group, as compared with the control group $(\mathrm{P}<0.05)$. In the PAH group, the $\varepsilon$ and SR of the middle and apical segments were significantly lower compared with basal segments $(\mathrm{P}<0.01)$. The SR of the apical segments were positively correlated with the area and diameter of the right ventricular. The $\varepsilon$ of the apical segments were positively correlated with RV area, diameter and systolic function. The $\varepsilon$ and SR of apical segments were positively correlated with pulmonary artery pressure and pulmonary vascular resistance measured by right heart catheterization. These findings indicated that 2DS can accurately evaluate RV longitudinal systolic function.
\end{abstract}

\section{Introduction}

Pulmonary arterial hypertension (PAH) is an increase of blood pressure in the pulmonary artery, with resting mean pulmonary arterial pressure $(\mathrm{mPAP}) \geq 25 \mathrm{mmHg}$. This can lead to increased lung vascular resistance, decreased right ventricular function, right heart failure and fatality (1). PAH has high disability and mortality rates, which pose a threat

Correspondence to: Dr Zhe Wen, Department of Ultrasound, Beijing Shijitan Hospital, Capital Medical University, 10 Tieyi Road, Beijing 100038, P.R. China

E-mail: 13520736069@163.com

Key words: two-dimensional strain echocardiography, pulmonary hypertension, right ventricular function to patient health and have a notable impact on a country's economy $(2,3)$.

The complex shape and volume dependency of the right ventricle (RV) make it difficult to accurately quantify $R V$ morphology and function using standard echocardiography $(4,5)$. Two-dimensional strain echocardiography (2DS) is based on strain $(\varepsilon)$ and strain rate (SR) imaging (6), and measures motion by tracking speckles. These speckles are natural acoustic markers, which are evenly and stably distributed in myocardial tissue, and move synchronously with the tissue. The speckles can be tracked in successive time frames of ultrasound imaging, and their movement can then be used to calculate the tissue's velocity, $\varepsilon$ and SR (7-9). The muscle fibers of the RV predominantly run in a longitudinal direction and the major contractility of RV occurs in the longitudinal plane, which indicates that global longitudinal strain can represent RV function accurately (10). The 2DS method has been frequently used to assess left ventricle function (7). However, it has rarely been used to examine RV function.

The current study aimed to compare the systolic strain capacity of the RV free wall in PAH patients and healthy control patients using 2DS, and to investigate the effect of mPAP on right ventricular systolic strain.

\section{Subjects and methods}

Subjects. A total of $41 \mathrm{PAH}$ patients were recruited from Beijing Shijitan Hospital (Beijing, China) from April 2007 to February 2009. The inclusion criteria for PAH patients were as follows: i) No previous history of respiratory disease, cardiovascular disease or pulmonary embolism, ii) PAH diagnosis, based on right heart catheterization (RHC) results showing that resting mPAP $>25 \mathrm{mmHg}(1 \mathrm{mmHg}=0.133 \mathrm{kPa})$ and pulmonary capillary wedge pressure $<15 \mathrm{mmHg}$. The exclusion criterion was diagnosis with pulmonary-related hypertension other than PAH, such as left heart-associated PAH, respiratory-associated PAH, chronic thromboembolic PAH or mixed PAH, as defined by the 2003 World Health Organization PAH diagnostic criteria (7). All patients underwent routine chest $\mathrm{X}$-rays and high-resolution computed tomography scans. Patients with severe interstitial lung disease were excluded. A total of 40 healthy control patients, with no previous history of smoking, cardiovascular disease or respiratory disease, were also recruited. All PAH patients received RHC $72 \mathrm{~h}$ before 
and after echocardiography. Written and informed consent was obtained from every patient prior to the study. The study was approved by the ethics review board of Beijing Shijitan Hospital.

Procedures. All patients underwent RHC in a resting state, according to standard protocol (11). The cardiac output (CO) of patients with idiopathic PAH (IPAH) was measured by thermodilution, and the $\mathrm{CO}$ of patients with congenital heart disease (CHD)/PAH was measured using the Fick method (11).

2DS was conducted using a cardiac ultrasound imaging device (Philips iE33), QLAB 7.1 cardiovascular ultrasound quantification software (both Philips Medical Systems, Inc., Bothell, WA, USA) and DVD storage. Patients were in the left lateral position and in a resting state. The chest-lead electrocardiogram recorded the 2DS in the apical four-chamber view (frame rate, 70-90 per sec) for three cardiac cycles. QLAB software was used to analyze the 2DS and manually outline the right ventricular endocardial surface. Then QLAB generated the area of interest, adjusting its width to match the thickness of the ventricular wall. QLAB tracked myocardial motion in the area of interest and measured the systolic $\varepsilon$ and SR of basal, middle and apical segments. The endocardial border of RV was manually traced at systole and diastole. This was used to calculate the RV area. The change rate was also calculated.

Statistical analysis. SPSS 15.0 (SPSS, Inc., Chicago, IL, USA) was used for statistical analysis. Data are expressed as the mean \pm standard deviation. Comparisons between echocardiography results were performed using a t-test. Correlations between measurements of RV function by $2 \mathrm{DS}, \mathrm{RHC}$ and conventional ultrasound were evaluated using Pearson correlation analysis. $\mathrm{P}<0.05$ was considered to indicate a statistically significant result.

\section{Results}

Baseline characteristics and echocardiography. There were 41 patients in the PAH group (32 cases of IPAH and 9 cases of CHD-PAH) and 40 healthy patients in the control group. There were no significant differences in age, gender, height, body mass or left ventricular ejection fraction between the two groups (Table I). The RV area change rate and blood pressure were significantly lower in the $\mathrm{PAH}$ group as compared with the control group $(\mathrm{P}<0.01$ and $\mathrm{P}<0.05$, respectively; Table I). The diastolic and systolic area of RV and the tricuspid regurgitation velocity were significantly higher in the PAH group compared with the control group (all $\mathrm{P}<0.01$; Table I).

$\varepsilon$ and $S R$ of $R V$ free wall segments. In order to evaluate differences in RV parameters between the two groups, $\varepsilon$ and SR were detected and compared (Figs. 1 and 2). The $\varepsilon$ and SR of the basal, middle and apical segments of RV free wall were significantly lower in the PAH group compared with the control group $(\mathrm{P}<0.05$; Table II). In the control group, there were no significant differences in $\varepsilon$ and SR between each segment of RV free wall (Table II). In the PAH group, the $\varepsilon$ and SR of the middle and apical segments were significantly lower compared with the basal segments $(\mathrm{P}<0.05$; Table II).
These results indicated that patients with PAH had impaired RV systolic strain and strain rates, compared with controls.

Correlation between 2DS and conventional ultrasound measurements of $R V$ function. The correlations between RV function parameters measured by 2DS and conventional ultrasound are shown in Table III. The SRs of middle segments were negatively correlated with RV diameter and area $(\mathrm{P}<0.05)$, and the SRs of apical segments were positively correlated with RV area $(\mathrm{P}<0.05)$. No correlation was found between the SR of basal segments and conventional ultrasound measurements. The $\varepsilon$ of apical segments was positively correlated with tricuspid regurgitation flow rate, $\mathrm{RV}$ diameter and area and RV area change rate (all $\mathrm{P}<0.01$ ), as well as with left ventricular stroke volume $(\mathrm{P}<0.05)$. The $\varepsilon$ of middle and basal segments were negatively correlated with RV area change rate and left ventricular stroke volume (both $\mathrm{P}<0.05$ ), but no correlations were found with other conventional ultrasound measurements.

Correlation between $2 D S$ and RHC measurements of $R V$ function. The correlations between RV function parameters measured by 2DS and RHC are shown in Table IV. The SRs of apical segments were positively correlated with pulmonary artery pressure (diastolic, systolic and mean pressure; $\mathrm{P}<0.05$, $\mathrm{P}<0.01, \mathrm{P}<0.01$, respectively) and total pulmonary resistance $(\mathrm{P}<0.01)$, and negatively correlated with stroke volume $(\mathrm{P}<0.05)$. The $\varepsilon$ of apical segments were positively correlated with pulmonary artery pressure (diastolic, systolic and mean pressure) and total pulmonary resistance and negatively correlated with stroke volume (all $\mathrm{P}<0.01$ ). The $\varepsilon$ of middle segments were positively correlated with total pulmonary resistance $(\mathrm{P}<0.01)$, but no correlation was detected between other measured parameters.

\section{Discussion}

The anatomy of the RV results in it having low resistance and a high load capacity (12). In pulmonary hypertension, pulmonary vascular resistance increases, resulting in increased RV afterload, RV hypertrophy and myocardial oxygen demand. It can also result in an insufficient blood supply, decreased RV compliance and increased RV diastolic pressure, leading to cavity dilation and potentially right heart failure (13-16).

The RV is principally composed of longitudinal muscle (17). Ejection is performed by longitudinal muscle, so the majority of RV function research has focused on longitudinal muscle (18-20). $\varepsilon$ and SR are indicators of cardiac function based on myocardial deformation. In the current study, 2DS was used to track speckles that were evenly distributed in myocardium in two-dimensional gray-scale images over multiple time frames. Movement of the speckles was tracked and their relative movement compared between time frames. This method overcomes angle dependence, so that RV longitudinal motion can be quantitatively recorded (8).

The current study demonstrated that SR and $\varepsilon$ were lower in each segment of RV in PAH patients compared with the control group, which is consistent with previous MRI findings (21). Results of the current study also demonstrated that $\mathrm{SR}$ and $\varepsilon$ in the apical and middle segments in PAH patients 
Table I. Baseline characteristics and echocardiography results of PAH and control groups.

\begin{tabular}{lcc}
\hline Parameter & Control $(\mathrm{n}=40)$ & PAH $(\mathrm{n}=41)$ \\
\hline Gender (male/female) & $36 / 4$ & $36 / 5$ \\
Age (years) & $31.8 \pm 17.2$ & $30.8 \pm 16.2$ \\
Brachial artery systolic pressure $(\mathrm{mmHg})$ & $119 \pm 13$ & $105 \pm 16^{\mathrm{a}}$ \\
Brachial artery diastolic pressure $(\mathrm{mmHg})$ & $75 \pm 16$ & $55 \pm 9^{\mathrm{a}}$ \\
Tricuspid regurgitation velocity $(\mathrm{m} / \mathrm{sec})$ & $1.9 \pm 0.6$ & $4.5 \pm 2.3^{\mathrm{b}}$ \\
RV diastolic area $\left(\mathrm{cm}^{2}\right)$ & $15.1 \pm 6.0$ & $33.1 \pm 13.9^{\mathrm{b}}$ \\
RV systolic area $\left(\mathrm{cm}^{2}\right)$ & $6.9 \pm 4.0$ & $23.3 \pm 10.9^{\mathrm{b}}$ \\
Area change rate of RV $(\%)$ & $50.9 \pm 10.8$ & $20.9 \pm 10.8^{\mathrm{b}}$ \\
Left ventricular ejection fraction $(\%)$ & $65.3 \pm 12.8$ & $70.3 \pm 15.8$
\end{tabular}

${ }^{\mathrm{a}} \mathrm{P}<0.05$ vs. control, ${ }^{\mathrm{b}} \mathrm{P}<0.01$ vs. control. $\mathrm{PAH}$, pulmonary arterial hypertension; $\mathrm{RV}$, right ventricle.

Table II. Comparison of $\varepsilon$ and SR between the PAH and control groups.

\begin{tabular}{llccc}
\hline & \multicolumn{2}{c}{ SR (sec-1) } & & $\varepsilon$ \\
\cline { 2 - 5 } Segment & PAH $(\mathrm{n}=41)$ & Control $(\mathrm{n}=40)$ & PAH $(\mathrm{n}=41)$ & Control $(\mathrm{n}=40)$ \\
\hline Basal & $-1.61 \pm 0.90^{\mathrm{a}}$ & $-2.03 \pm 1.19$ & $-23.2 \pm 10.6^{\mathrm{a}}$ & $-43.2 \pm 12.6$ \\
Middle & $-1.41 \pm 0.64^{\mathrm{a}, \mathrm{b}}$ & $-1.79 \pm 0.69$ & $-17.2 \pm 5.0^{\mathrm{a}, \mathrm{b}}$ & $-32.2 \pm 8.3$ \\
Apical & $-1.22 \pm 0.56^{\mathrm{a}, \mathrm{b}}$ & $-1.69 \pm 0.59$ & $-18.7 \pm 6.9^{\mathrm{a}, \mathrm{b}}$ & $-34.2 \pm 9.4$ \\
\hline
\end{tabular}

${ }^{\mathrm{a}} \mathrm{P}<0.05$ vs. control, ${ }^{\mathrm{b}} \mathrm{P}<0.05$ vs. basal segment. $\mathrm{PAH}$, pulmonary arterial hypertension; $\mathrm{SR}$, strain rate; $\varepsilon$, strain.
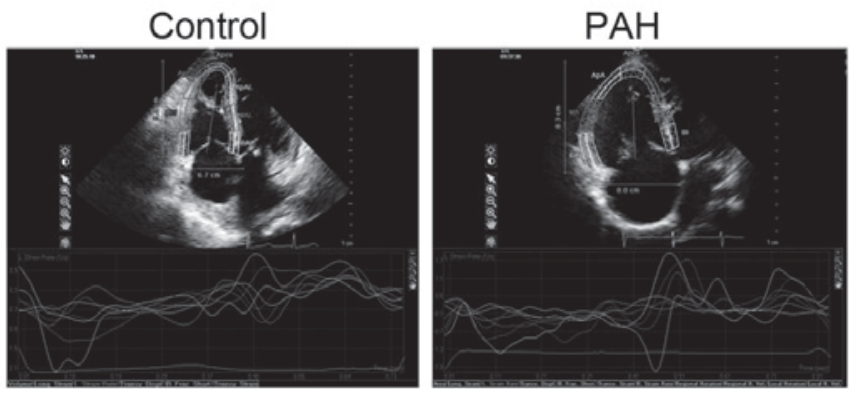

Figure 1. RV longitudinal $\varepsilon$ determined by 2D speckle tracking echocardiography in control and PAH groups. The RV wall was divided into six segments automatically. Peak longitudinal $\varepsilon$ was measured in each segment at the mid-RV free wall and interventricular septum. RV, right ventricular; $\mathrm{PAH}$, pulmonary arterial hypertension; $\varepsilon$, strain.

were significantly lower compared with basal segments. Apical segment deformation was correlated with RV contraction measured by RHC and conventional ultrasound, suggesting that under long-term pressure, the level of damage in the RV apical segment was higher than in the RV basal segment. This is likely to be due to the anatomy of the basal segment, which is composed of smooth myocardial and more longitudinal muscle, while the apical segment is composed of trabecular and less longitudinal muscle $(22,23)$. This means that the apical segment is more easily damaged than the basal segment under the same pressure $(24,25)$. Therefore, this suggests that
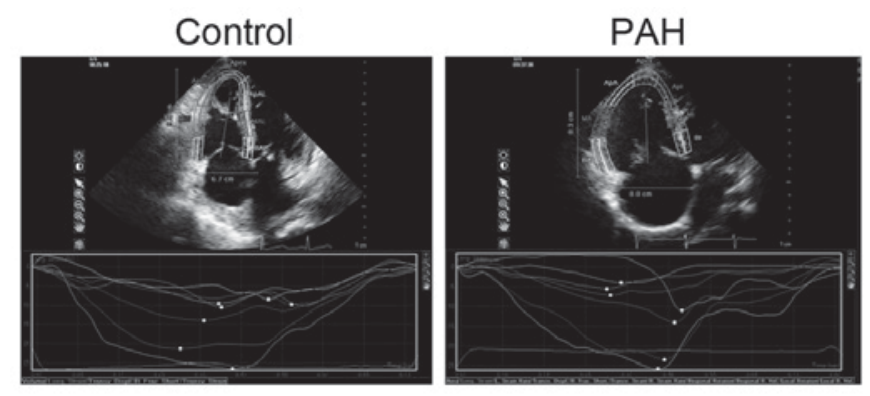

Figure 2. RV longitudinal strain rate determined by 2D speckle tracking echocardiography in control and PAH groups. The RV wall was divided into six segments automatically. Peak longitudinal strain was measured in each segment at the mid-RV free wall and interventricular septum. RV, right ventricular; PAH, pulmonary arterial hypertension.

$\varepsilon$ and SR of the apical segment are important in determining the extent of PAH.

It has previously been reported that the basal segment is more severely damaged than the apical segment in PAH patients, which is inconsistent with the results of the current study $(22,23)$. This is likely to be due to the patients included in the studies. Only IPAH and CHD-PAH patients were included in the current study, while respiratory disease-associated PAH and thromboembolic PAH were also included in previous studies. It has also previously been suggested that the reverse McConnell sign in thromboembolic PAH patients can affect 
Table III. Correlation between two-dimensional strain echocardiography and conventional ultrasound measurements of RV deformation in pulmonary arterial hypertension patients $(n=41)$.

\begin{tabular}{|c|c|c|c|c|c|c|}
\hline \multirow[b]{2}{*}{ Parameter } & \multicolumn{2}{|c|}{ Basal } & \multicolumn{2}{|c|}{ Middle } & \multicolumn{2}{|c|}{ Apical } \\
\hline & SR r & $\varepsilon \mathrm{r}$ & SR r & $\varepsilon \mathrm{r}$ & SR r & $\varepsilon \mathrm{r}$ \\
\hline Tricuspid regurgitation peak velocity & -0.22 & 0.25 & -0.32 & 0.25 & 0.36 & $0.62^{\mathrm{b}}$ \\
\hline RV diameter & -0.17 & 0.18 & $-0.47^{\mathrm{a}}$ & 0.18 & 0.31 & $0.52^{\mathrm{b}}$ \\
\hline RV diastolic area & -0.23 & 0.35 & $-0.43^{\mathrm{a}}$ & 0.35 & $0.41^{\mathrm{a}}$ & $0.68^{\mathrm{b}}$ \\
\hline RV systolic area & -0.20 & 0.26 & $-0.52^{\mathrm{a}}$ & 0.26 & $0.41^{\mathrm{a}}$ & $0.64^{\mathrm{b}}$ \\
\hline $\mathrm{RV}$ area change rate & -0.18 & $-0.58^{\mathrm{a}}$ & -0.38 & $-0.58^{\mathrm{a}}$ & -0.34 & $0.70^{\mathrm{b}}$ \\
\hline Left ventricular stroke volume & 0.08 & $-0.42^{\mathrm{a}}$ & 0.08 & $-0.52^{\mathrm{a}}$ & -0.25 & $0.40^{\mathrm{a}}$ \\
\hline
\end{tabular}

${ }^{\mathrm{a}} \mathrm{P}<0.05,{ }^{\mathrm{b}} \mathrm{P}<0.01$. RV, right ventricle; $\mathrm{r}$, correlation coefficient; $\mathrm{SR}$, strain rate; $\varepsilon$, strain.

Table IV. Correlation between two-dimensional strain echocardiography and right heart catheterization measurements of RV deformation in pulmonary arterial hypertension patients $(n=41)$.

\begin{tabular}{|c|c|c|c|c|c|c|c|}
\hline \multirow[b]{2}{*}{ Parameter } & \multirow[b]{2}{*}{ Value } & \multicolumn{2}{|c|}{ Basal } & \multicolumn{2}{|c|}{ Middle } & \multicolumn{2}{|c|}{ Apical } \\
\hline & & SR r & $\varepsilon \mathrm{r}$ & SR r & $\varepsilon \mathrm{r}$ & SR r & $\varepsilon \mathrm{r}$ \\
\hline Pulmonary artery systolic pressure $(\mathrm{mmHg})$ & $73 \pm 39$ & 0.08 & 0.40 & 0.19 & 0.41 & $0.59^{\mathrm{a}}$ & $0.77^{\mathrm{b}}$ \\
\hline Pulmonary artery diastolic pressure (mmHg) & $29 \pm 12$ & -0.16 & 0.38 & 0.21 & 0.42 & $0.68^{b}$ & $0.82^{\mathrm{b}}$ \\
\hline Mean pulmonary artery pressure (mmHg) & $49 \pm 16$ & -0.09 & 0.44 & 0.19 & 0.40 & $0.69^{\mathrm{b}}$ & $0.79^{b}$ \\
\hline Stroke volume $(\mathrm{ml})$ & $55 \pm 16$ & 0.12 & -0.41 & 0.02 & 0.38 & $-0.54^{\mathrm{a}}$ & $-0.59^{b}$ \\
\hline Total pulmonary resistance (Wood units) & $95 \pm 50$ & 0.02 & 0.48 & 0.26 & $0.60^{\mathrm{b}}$ & $0.65^{\mathrm{b}}$ & $0.77^{\mathrm{b}}$ \\
\hline
\end{tabular}

${ }^{\mathrm{a}} \mathrm{P}<0.05,{ }^{\mathrm{b}} \mathrm{P}<0.01$. r, correlation coefficient; $\mathrm{SR}$, strain rate; $\varepsilon$, strain.

study results (16). Moreover, the aforementioned studies used ultrasound estimation to evaluate RV function and hemodynamics, while RHC was used for evaluation in the current study. It has been reported in a meta-analysis that conventional ultrasound cannot accurately evaluate the extent of PAH or systolic function (13-15), due to the irregular shape of the $\mathrm{RV}$ and technical limitations of ultrasound in measuring area change rate and ejection fraction.

A limitation of the current study is that only IPAH and CHD-PAH were included, thus no information on the impact and differentiation of other types of PAH on the RV has been provided. Further studies involving additional PAH types are required.

In conclusion, the longitudinal function of RV in $\mathrm{PAH}$ patients was demonstrated to be significantly lower compared with the control group, according to SR and $\varepsilon$ measurements made using 2DS. The longitudinal function was found to be most significantly reduced in the apical segment. Thus, 2DS is able to accurately evaluate RV longitudinal systolic function.

\section{Acknowledgements}

This study was supported by the Capital Health Research and Development Special Foundation of China (grant no. 2014-4-2082), the Basic Clinical Research Cooperation
Project of Capital Medical University (grant no. 14JL87) and the Beijing Municipal Administration of Hospitals Incubating Program (grant no. PX2016023).

\section{References}

1. Rubin LJ: Primary pulmonary hypertension. N Engl J Med 336: 111-117, 1997

2. Oxborough D and Lord R: Predicting mortality in pulmonary arterial hypertension: Can it really be that simple? JACC Cardiovasc Imaging 8: 639-641, 2015.

3. Gu S, Hu Hand Dong H: Systematic review of the economic burden of pulmonary arterial hypertension. Pharmacoeconomics 34 : 533-550, 2016

4. Ploegstra MJ, Roofthooft MT, Douwes JM, Bartelds B, Elzenga NJ, van de Weerd D, Hillege HL and Berger RM: Echocardiography in pediatric pulmonary arterial hypertension: Early study on assessing disease severity and predicting outcome. Circ Cardiovasc Imaging 8: pii: e000878, 2014.

5. Haddad F, Hunt SA, Rosenthal DN and Murphy DJ: Right ventricular function in cardiovascular disease, part I: Anatomy, physiology, aging, and functional assessment of the right ventricle. Circulation 117: 1436-1448, 2008.

6. Pedrinelli R, Canale ML, Giannini C, Talini E, Dell'Omo G and Di Bello V: Abnormal right ventricular mechanics in early systemic hypertension: A two-dimensional strain imaging study. Eur J Echocardiogr 11: 738-742, 2010.

7. Woo JS, Yu TK, Kim WS, Kim KS and Kim W: Early prediction of myocardial viability after acute myocardial infarction by two-dimensional speckle tracking imaging. J Geriatr Cardiol 12: 474-481, 2015. 
8. Luo AG, Yin LX, Chun-Mei LI, Wang LC, Lin JD, Cai YL and Meng QG: Measurement of left ventricular torsion with cardiac pacing and right bundle branch block patients by two-dimensional ultrasound speckle tracking imaging. Chinese J Ultrasonography: 15, 2006.

9. Meluzín J, Spinarová L, Bakala J, Toman J, Krejcí J, Hude P, Kára T and Soucek M: Pulsed Doppler tissue imaging of the velocity of tricuspid annular systolic motion; a new, rapid and non-invasive method of evaluating right ventricular systolic function. Eur Heart J 22: 340-348, 2001.

10. Pirat B, McCulloch ML and Zoghbi WA: Evaluation of global and regional right ventricular systolic function in patients with pulmonary hypertension using a novel speckle tracking method. Am J Cardiol 98: 699-704, 2006.

11. Rosenkranz S and Preston IR: Right heart catheterisation: Best practice and pitfalls in pulmonary hypertension. Eur Respir Rev 24: 642-652, 2015.

12. Bradlow WM, Gibbs JS and Mohiaddin RH: Cardiovascular magnetic resonance in pulmonary hypertension. J Cardiovasc Magn Reson 14: 6, 2012.

13. Xie Y, Burke BM, Kopelnik A, Auger W, Daniels LB Madani MM, Poch DS, Kim NH and Blanchard DG: Echocardiographic estimation of pulmonary vascular resistance in chronic thromboembolic pulmonary hypertension: Utility of right heart Doppler measurements. Echocardiography 31: 29-33, 2014.

14. Haghi D, Sueselbeck T, Poerner T, Borggrefe M and Haase KK: A novel regional right ventricular wall-motion abnormality observed in a case of acute pulmonary embolism (reverse McConnell sign). J Am Soc Echocardiogr 18: 75-77, 2005.

15. Aggeli C, Lagoudakou S, Felekos I, Panagopoulou V, Kastellanos S, Toutouzas K, Roussakis G and Tousoulis D: Two-dimensional speckle tracking for the assessment of coronary artery disease during dobutamine stress echo: Clinical tool or merely research method. Cardiovasc Ultrasound 13: 43, 2015.
16. Bossone E, Dellegrottaglie S, Patel S, Grunig E, D'Andrea A, Ferrara F, Gargiulo P, D'Alto M, Soricelli A, Cittadini A, et al: Multimodality imaging in pulmonary hypertension. Can J Cardiol 31: 440-459, 2015.

17. Forfia PR and Vachiéry JL: Echocardiography in pulmonary arterial hypertension. Am J Cardiol 110 (6 Suppl): 16S-24S, 2012.

18. Multicenter Postinfarction Research Group: Risk stratification and survival after myocardial infarction. N Engl J Med 309: 331-336, 1983.

19. Biancari F, Vasques F, Mikkola R, Martin M, Lahtinen J and Heikkinen J: Validation of EuroSCORE II in patients undergoing coronary artery bypass surgery. Ann Thorac Surg 93: 1930-1935, 2012.

20. Lopez-Candales A and Edelman K: Shape of the right ventricular outflow Doppler envelope and severity of pulmonary hypertension. Eur Heart J Cardiovasc Imaging 13: 309-316, 2012

21. Kaul S, Tei C, Hopkins JM and Shah PM: Assessment of right ventricular function using two-dimensional echocardiography. Am Heart J 107: 526-531, 1984.

22. Cresci SG and Goldstein JA: Hemodynamic manifestations of ischemic right heart dysfunction. Cathet Cardiovasc Diagn 27: 28-34, 1992.

23. Schenk P, Globits S, Koller J, Brunner C, Artemiou O, Klepetko W and Burghuber OC: Accuracy of echocardiographic right ventricular parameters in patients with different end-stage lung diseases prior to lung transplantation. J Heart Lung Transplant 19: 145-154, 2000.

24. Italia LJ: Anatomy and physiology of the right ventricle. Cardiol Clin 30: 167-187, 2012.

25. Jiang B, Deng Y, Suen C, Taha M, Chaudhary KR, Courtman DW and Stewart DJ: Marked strain-specific differences in the SU5416 rat model of severe pulmonary arterial hypertension. Am J Respir Cell Mol Biol 54: 461-468, 2016. 